\title{
Conchoid surfaces of rational ruled surfaces
}

\author{
Martin Peternell, David Gruber, Juana Sendra
}

November 13, 2010

\begin{abstract}
The conchoid of a surface $F$ with respect to given fixed point $O$ is roughly speaking the surface obtained by increasing the radius function with respect to $O$ by a constant. This paper studies real rational ruled surfaces in this context and proves that their conchoids possess real rational parameterizations, independently on the focus. Thus any rational ruled surface $F$ admits a rational radius function $r(u, v)$ with respect to any point in space. By increasing this radius function to $r(u, v)+d$, the conchoids of $F$ are represented in an analogous way. Besides the general skew ruled surfaces and examples of low algebraic degree we study rational cylinders, cones, and tangent surfaces of rational space curves.
\end{abstract}

Keywords: rational ruled surface, rational conchoid surface, polar representation, rational radius function, pencil of conics.

\section{Introduction}

The conchoid is a classical geometric construction and dates back already to the ancient Greeks. Given a planar curve $C$, a fixed point $O$ and a constant distance $d$, the conchoid $D$ of $C$ with respect to $O$ at distance $d$ is the (Zariski closure of the) set of points $Q$ in the line $O P$ at distance $d$ of a moving point $P$ varying in the curve $C$,

$$
D=\{Q \in O P \text { with } P \in C \text {, and } \overline{Q P}=d\}^{*},
$$

where the asterisk denotes the Zariski closure; for a formal definition of conchoid in terms of diagrams of incidence we refer to $[12,13]$. The definition of the conchoid surface to a given surface $F$ in space with respect to a given point $O$ and distance $d$ follows analogous lines. Note that the definition of the conchoid (either for curves or for surfaces) by means of diagrams of incidence, in combination with the Closure Theorem (see [3] p. 122), imply 
that elimination theory techniques, such as Gröbner bases, provide the equations of the conchoid.

We briefly turn to the curve case to discuss some important properties. For an analytic representation it is convenient to choose $O=(0,0)$. Using a representation of a curve $C$ in terms of polar coordinates $\mathbf{c}(t)=r(t)(\cos t, \sin t)$, its conchoid $D$ with respect to $O$ and distance $d$ is obtained by $\mathbf{d}(t)=(r(t) \pm d)(\cos t, \sin t)$. More generally we can consider any parameterization $\mathbf{k}(t)$ of the unit circle $S^{1}$. The curve $C$ and its conchoids $D$ are represented by

$$
\mathbf{c}(t)=r(t) \mathbf{k}(t) \text { and } \mathbf{d}(t)=(r(t) \pm d) \mathbf{k}(t), \text { with }\|\mathbf{k}\|=1
$$

Surfaces and their conchoids are analogously represented. With respect to a given point $O=(0,0,0)$ and a parameterization $\mathbf{k}(u, v)$ of the unit sphere $S^{2}$, a surface $F$ is parameterized by $\mathbf{f}(u, v)=r(u, v) \mathbf{k}(u, v)$. The conchoid surface $G$ of $F$ and distance $d$ is represented by

$$
\mathbf{g}(u, v)=(r \pm d) \mathbf{k}(u, v) .
$$

We consider trigonometric parameterizations $\mathbf{k}=(\cos u \cos v, \sin u \cos v, \sin v)$ of $S^{2}$ or rational ones, for instance $\mathbf{k}=\left(2 a c, 2 b c, a^{2}+b^{2}-c^{2}\right) /\left(a^{2}+b^{2}+c^{2}\right)$. This rational parameterization originates from a stereographic projection with center $(0,0,-1)$ which maps points $(a / c, b / c, 0)$ of the plane $z=0$ to points $\mathbf{k}$ in $S^{2}$. Here, $a, b$ and $c$ can be considered as polynomials; often used is $a=u, b=v$ and $c=1$. More generally the unit sphere $S^{2}$ admits rational parameterizations $\mathbf{k}=(A / D, B / D, C / D)$, with

$$
A=2(a c+b d), B=2(b c-a d), C=a^{2}+b^{2}-c^{2}-d^{2}, D=a^{2}+b^{2}+c^{2}+d^{2},
$$

where $a, b, c$ and $d$ are assumed to be polynomials in $u$ and $v$.

Conchoids of curves can be found in several monographs on curves and surfaces, see for instance [5]. The classical conchoid of Nicomedes, that is the conchoid of a line, appeared already 200 B.C. The conchoid of a circle with respect to one of its points is also a well known example, called Limacon of Pascal. A recent careful investigation of algebraic properties of conchoid curves and conditions for their rationality, as well as direct parameterization algorithms, can be found in $[12,13]$.

The conchoid of a curve can be considered as special case of the cissoid of two curves. Given two curves $A$ and $B$ and a fixed point $O$, and let $P$ and $Q$ be two points in $A$ and $B$, respectively, which are collinear with $O$. The cissoid of $A$ and $B$ with respect to $O$ is the set of points $X$ in the lines $O P$ for $P \in A$ which satisfy the relation $\overline{O X}=\overline{O P}-\overline{O Q}$. By specializing $B$ as unit circle centered at $O$, we arrive at the definition of the conchoid of $A$ with respect to $O$. Some authors use the sum instead of the difference in the cissoid's definition. By reflecting $B$ at $O$ these definitions are equivalent. A recent publication dealing with this topic is [2]. 
Contribution: The aim of this contribution is to study rational ruled surfaces and their conchoids. Without loss of generality we choose $O=(0,0,0)$ when not defined in another way. The main result being proved is that any rational ruled surface $F$ admits a rational polar representation $\mathbf{f}(u, v)=r(u, v) \mathbf{k}(u, v)$ with a rational radius function $r(u, v)$ and a particular rational parameterization $\mathbf{k}(u, v)$ of the unit sphere $S^{2}$. This implies that the conchoids $G$ of $F$ with respect to any focus in $\mathbb{R}^{3}$ admit rational parameterizations. In general it is not true that the behavior of the rationality is independent of the focus, see for instance section 5 in [13] (for the case of curves) and example 3.6 (for the case of surfaces).

The construction of $r(u, v) \mathbf{k}(u, v)$ involves finding a suitable rational parameterization $\mathbf{k}(u, v)$ of $S^{2}$ for a given rational ruled surface $F$ together with the determination of the radius function $r(u, v)$. In the general case this amounts in parameterizing a rational one-parameter family of conics in $\mathbb{P}^{2}$, see Section 2.

We investigate this general case as well as several particular cases where the parameterization problem turns out to be simpler. Rational cones and cylinders are studied in Sections 2.5 and 2.4, rational rotational ruled surfaces in Section 2.6 and special cases are dicussed in Sections 2.3 and 2.7. Geometric considerations and arguments go along with this discussion. Further we give a couple of examples in Section 3 to illustrate the results in a better way.

\subsection{Local properties of conchoid surfaces}

Given a surface $\mathbf{f}(u, v)=r(u, v) \mathbf{k}(u, v)$, with $\|\mathbf{k}\|=1$. ¿From this polar representation we can derive a local property concerning the path normals of the conchoids surfaces for varying distance $d$ and fixed parameter values $u$ and $v$. This can also be obtained considering the ray emanating from $O=(0,0,0)$ as a moving system where the surface point $\mathbf{f}(u, v)$ is sliding on the ray as well as on the surface $F$ itself.

Let $\mathbf{k}(u, v)$ be a parameterization of the unit sphere $S^{2}$ such that $\mathbf{k}, \mathbf{k}_{u}, \mathbf{k}_{v}$ are an orthonormal basis in $\mathbb{R}^{3}$. We compute the partial derivatives of the conchoid $\mathbf{g}=(r+d) \mathbf{k}$, and obtain

$$
\mathbf{g}_{u}=(r+d) \mathbf{k}_{u}+r_{u} \mathbf{k} \text {, and } \mathbf{g}_{v}=(r+d) \mathbf{k}_{v}+r_{v} \mathbf{k} .
$$

Taking the relations $\mathbf{k} \times \mathbf{k}_{u}=\mathbf{k}_{v}$ and $\mathbf{k}_{v} \times \mathbf{k}=\mathbf{k}_{u}$ into account, a normal vector of $\mathbf{g}$ reads $\mathbf{n}=-(r+d) \mathbf{k}+r_{u} \mathbf{k}_{u}+r_{v} \mathbf{k}_{v}$. Thus, all the normals of the conchoids for fixed position $\mathbf{k}$ and varying $d$ pass through the point $\mathbf{v}=r_{u} \mathbf{k}_{u}+r_{v} \mathbf{k}_{v}$, being the intersection of the normal through $\mathbf{g}$ with the plane $\mathbf{k} \cdot \mathbf{x}=0$. This plane passes through $O$ and is parallel to the tangent plane to $S^{2}$ at $\mathbf{k}$.

The tangent planes to all conchoid surfaces for a fixed radius line $\lambda \mathbf{k}$ envelope a parabolic cylinder $Z$. The cross section parabola $P$ which is perpendicular to the generating lines of $Z$ has $\mathbf{v}$ as focal point and $O$ as vertex, see Fig. 1. 

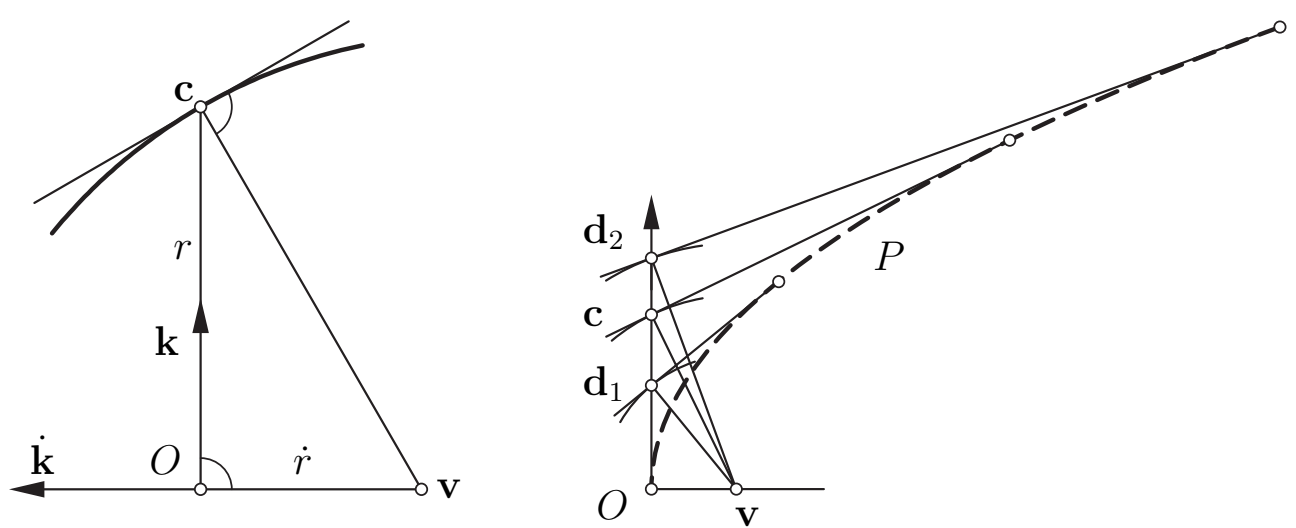

Figure 1: Left: Instantaneous center of rotation. Right: Parabola $P$ as envelope of the tangents to conchoids for varying distance.

\subsection{Transformations and conchoid curves}

To keep things simple we consider the curve case but the following arguments easily carry over to conchoids of surfaces. Let a curve $C$ in $\mathbb{R}^{2}$ be represented in polar coordinates by $\mathbf{c}(t)=r(t) \mathbf{k}(t)$, with $\|\mathbf{k}\|=1$. Applying a rotation $\rho: \mathbf{x}^{\prime}=R \cdot \mathbf{x}$ with a $2 \mathrm{x} 2$ rotation matrix $R$ maps $C$ to $C^{\prime}$, and the conchoids of $C$ are obviously mapped to conchoids of $C^{\prime}$ for respective distances.

If we apply a Euclidean displacement whose rotational part has a center different from the focus point, we have to map not only the curves but also the focus point for the conchoid construction to obtain invariance. This is easily seen by considering a pure translation. For a curve $C$ having a rational representation $\mathbf{c}(t)=r(t) \mathbf{k}(t)$ with respect to the origin $O=(0,0)$, its polar representation with respect to any other point is typically not rational.

In what follows we often use normal forms for surface representations. With respect to the arguments just outlined we may apply rotations and central similarities with respect to the origin to simplify the surface representation. The conchoid surfaces are always constructed with respect to the origin $O=(0,0,0)$. Later we prove that the conchoid surfaces of rational ruled surfaces are rational, independently of the chosen point $O$. This is quite surprising, since in the plane curve case this is not true in general (see Section 5 in [13]).

To introduce to the subject of conchoid curves and surfaces, we deal with two first simple examples, the conchoid of a line in $\mathbb{R}^{2}$ and the conchoid of a plane in $\mathbb{R}^{3}$, where the first example gives the conchoid of Nicomedes and has even been studied by the ancient Greeks. We introduce to basic techniques to construct parameterizations of conchoids.

Typically we always use Cartesian coordinates $(x, y)$ to represent points in $\mathbb{R}^{2}$. For representing ideal points in the projective plane $\mathbb{P}^{2} \supset \mathbb{R}^{2}$ we use homogeneous coordinates $\left(x_{0}, x_{1}, x_{2}\right) \mathbb{R}$, which are determined only up to a common factor. Assuming $x_{0}=0$ to be 

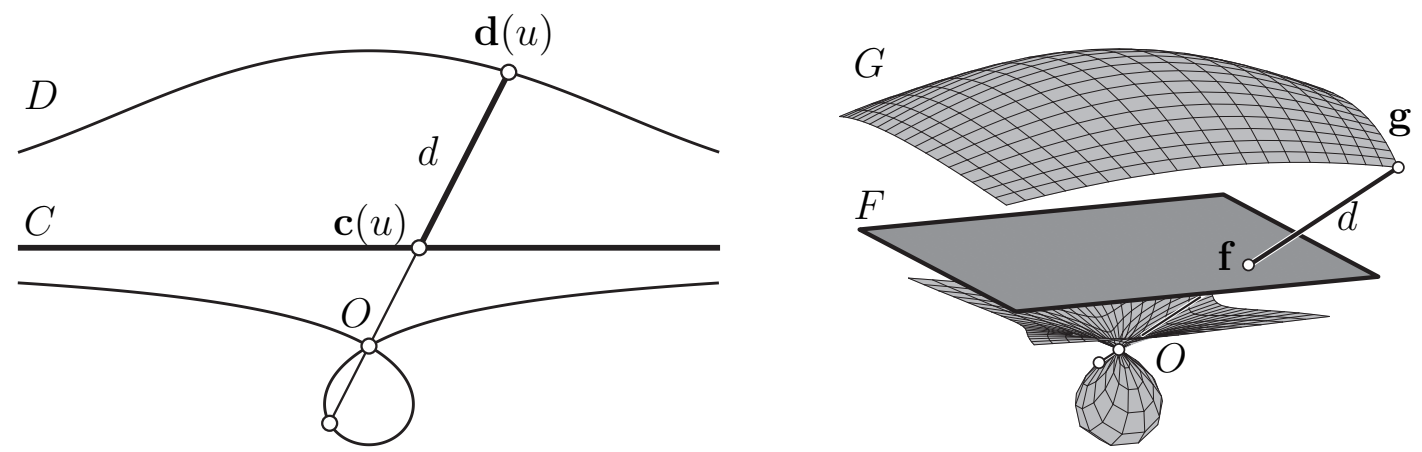

Figure 2: Left: Conchoid of a line in $\mathbb{R}^{2}$. Right: Conchoid of a plane in $\mathbb{R}^{3}$.

the ideal line, the conversion between Cartesian and homogeneous coordinates is $x=x_{1} / x_{0}$ and $y=x_{2} / x_{0}$. Analogously $(x, y, z)$ and $\left(x_{0}, x_{1}, x_{2}, x_{3}\right) \mathbb{R}$ are Cartesian and homogeneous coordinates in $\mathbb{R}^{3}$ and $\mathbb{P}^{3}$, respectively.

\subsection{The conchoid of a line in $\mathbb{R}^{2}$ and a plane $\mathbb{R}^{3}$}

Since the conchoid construction is invariant with respect to rotations and central similarities, we may assume that the line $C$, whose conchoid we want to construct, is given by $y=1$. Let $\mathbf{k}(u, v)=(\cos u, \sin u)$ be a parameterization of the unit circle, then $C: y=1$ is obtained for the radius function $r(u)=1 / \sin (u)$. In detail this reads

$$
\mathbf{c}(u)=\frac{1}{\sin u}(\cos u, \sin u)=\left(\frac{\cos u}{\sin u}, 1\right) .
$$

Increasing $r(u)$ by a constant $d$ leads to a trigonometric parameterization of the conchoid $D$ of $C$,

$$
\mathbf{d}(u)=\frac{1+d \sin u}{\sin u}(\cos u, \sin u) .
$$

Substituting trigonometric functions by rational functions converts (4) and (5) into rational parameterizations of $C$ and $D$. By eliminating the parameter $u$ we find that $D$ is an algebraic curve of order four,

$$
D: y^{2}\left(x^{2}+y^{2}\right)-2 y\left(x^{2}+y^{2}\right)+x^{2}+y^{2}\left(1-d^{2}\right)=0 .
$$

Since this equation does not contain constant and linear terms in $x$ and $y$, the origin $O=(0,0)$ is a double point of $D$. Moreover the leading term is $y^{2}\left(x^{2}+y^{2}\right)$, thus $D$ is circular which means that $D$ passes through the ideal points $(0,1, i) \mathbb{R}$ and $(0,1,-i) \mathbb{R}$, besides the ideal double point at $(0,1,0) \mathbb{R}$.

In an analogous way one finds a parameterization of the conchoid surfaces of a plane $F$ in $\mathbb{R}^{3}$. Applying a rotation and a scaling with center $O=(0,0,0)$, we assume $F: z=1$. 
Considering the parameterization $\mathbf{k}(u, v)=(\cos u \cos v, \sin u \cos v, \sin v)$ of the unit sphere, the plane $F: z=1$ is represented by the radius function $r(u, v)=1 / \sin v$. Thus the conchoid surface $G$ of the plane $F$ admits the trigonometric parameterization

$$
\mathbf{g}(u, v)=(r(u, v)+d) \mathbf{k}(u, v)=\frac{1+d \sin v}{\sin v}(\cos u \cos v, \sin u \cos v, \sin v),
$$

and an implicit equation of $G$ reads

$$
G: z^{2}\left(x^{2}+y^{2}+z^{2}\right)-2 z\left(x^{2}+y^{2}+z^{2}\right)+x^{2}+y^{2}+z^{2}\left(1-d^{2}\right)=0 .
$$

The intersection of $G$ with the ideal plane is $z^{2}\left(x^{2}+y^{2}+z^{2}\right)=0$ and consists of the ideal conic $x^{2}+y^{2}+z^{2}=0$ and the doubly counted line $z^{2}=0$. The conchoid $G$ is a surface of rotation of degree four with $z$ as axis. The horizontal intersections are circles with centers on $z$ and $G$ can be generated by rotating the conchoid of a line around $z$. the generating curve of $G$ is a conchoid of a line.

Considering the plane $F$ in general position with respect to the coordinate system, the unknown radius function of the plane $F$ can also be obtained by inserting the parameterization $r(u, v) \mathbf{k}(u, v)$ into the linear equation $F: \mathbf{a} \cdot \mathbf{x}+a_{0}=0$, with $a_{0} \neq 0$ and $\mathbf{a}=\left(a_{1}, a_{2}, a_{3}\right)$ as normal vector of $F$. This results in $r(u, v)=-a_{0} /(\mathbf{a} \cdot \mathbf{k})$. Planes passing through the origin $\left(a_{0}=0\right)$ have to be excluded here, since these planes are not in bijective correspondence to the bundle of lines with vertex $O=(0,0,0)$.

Considering quadrics passing through the origin $O$, the presented method to determine $r(u, v)$ works too. Let a quadric $F \ni O$ be given by the implicit equation $F: \mathbf{x}^{T} \cdot A$. $\mathbf{x}+\mathbf{b}^{T} \cdot \mathbf{x}=0$. Inserting a rational polar representation $r(u, v) \mathbf{k}(u, v)$ with an unknown function $r(u, v)$ into $F$ gives a rational solution $r(u, v)=-(\mathbf{b} \cdot \mathbf{k}) /\left(\mathbf{k}^{T} \cdot A \cdot \mathbf{k}\right)$ besides the trivial solution $r=0$. This argumentation can be extended to monomial algebraic surfaces of degree $n$ with an $n-1$-fold point at $O$ and it is proved easily that their conchoid surfaces are rational. Note that in this whole paragraph the focus is not taken generic but as a point on the quadric in the first case and as the singularity in the second case. Indeed, changing the focus (e.g out of the quadric) one may have different behaviors.

We summarize the presented parameterizations technique: to construct a parameterization of the conchoid surface $G$ of a given surface $F$ with respect to the origin $O$, we have to determine a polar representation $r(u, v) \mathbf{k}(u, v)$, where $\mathbf{k}(u, v)$ is a suitable parameterization of $S^{2}$. If both $r(u, v)$ and $\mathbf{k}(u, v)$ are rational functions, the surface $F$ has a rational polar representation and its conchoid surfaces $G$ are rational. The following sections contain a detailed discussion for rational ruled surfaces $F$ and their conchoids.

\section{The conchoids of rational ruled surfaces in $\mathbb{R}^{3}$}

A ruled surface $F$ carries a one-parameter family of straight lines, thus admits a parametric representation $\mathbf{f}(u, v)=\mathbf{c}(u)+v \mathbf{e}(u)$, where $\mathbf{c}(u)$ is called directrix curve and $\mathbf{e}(u)$ is a 
direction vector field of $F$ 's generating lines. The generating curve $\mathbf{c}$ on $F$ can be exchanged by any other curve $\mathbf{y}(u)=\mathbf{c}(u)+v(u) \mathbf{e}(u)$.

Let $\mathbf{f}_{u}=\dot{\mathbf{c}}+v \dot{\mathbf{e}}$ and $\mathbf{f}_{v}=\mathbf{e}$ be the partial derivatives of $\mathbf{f}$. The normal vector

$$
\mathbf{n}(u, v)=\dot{\mathbf{c}}(u) \times \mathbf{e}(u)+v \dot{\mathbf{e}}(u) \times \mathbf{e}(u)=\mathbf{n}_{1}(u)+v \mathbf{n}_{2}(u)
$$

is the linear combination of the vectors $\mathbf{n}_{1}$ and $\mathbf{n}_{2}$. Given a fixed generating line $L$ of $F$, the normal vectors along $L$ may be linearly independent or not. In the previous case $L$ is called skew generating line, and in case of linear dependece $L$ is called torsal generating line of $F$. A ruled surface carrying only a finite number of torsal generators is called skew ruled surface. If all generating lines of $F$ are torsal, $F$ is a developable ruled surface. Examples for the latter family are cylinders, cones and tangent surfaces of space curves.

We now investigate conchoid surfaces of rational ruled surfaces starting with the general case of skew ruled surfaces. It will turn out in Sections 2.4 and 2.5 that the construction for cylinders and cones is somehow simpler, but the general concept can be applied in any special case too.

\subsection{General construction of conchoids of ruled surfaces}

Let a ruled surface $F$ be given by $\mathbf{f}(u, v)=\mathbf{c}(u)+v \mathbf{e}(u)$. Typically it is difficult to define $F$ as zero set of an implicit function. In order to find a rational polar representation $r(u, v) \mathbf{k}(u, v)$ of $F$, with a rational radius function $r(u, v)$ with respect to the origin and some rational parameterization $\mathbf{k}(u, v)$ of $S^{2}$, we investigate the squared length

$$
\|\mathbf{f}(u, v)\|^{2}=\mathbf{f}(u, v)^{2}=\mathbf{c}(u)^{2}+2 v \mathbf{c}(u) \cdot \mathbf{e}(u)+v^{2} \mathbf{e}(u)^{2} .
$$

In the following we prove that there exists a reparameterization of $F$ such that $\|\mathbf{f}(u, v)\|$ is a rational function. The construction being presented constructs a curve $\mathbf{y}(u)=\mathbf{c}(u)+$ $v(u) \mathbf{e}(u)$ of $F$ with rational radius $\|\mathbf{y}(u)\|$ at first. In a second step we show how to extend this result to a rational polar representation of $F$. The constructive proof that any rational ruled surface $F$ carries a curve $\mathbf{y}(u)$ with rational radius function $\|\mathbf{y}(u)\|$ mainly uses on the following lemma.

Lemma 1 Let $A(u)$ be a one parameter family of conics in $\mathbb{P}^{2}$ given by the equation

$$
A(u):\left(x_{0}, x_{1}, x_{2}\right) \cdot A(u) \cdot\left(x_{0}, x_{1}, x_{2}\right)^{T}=0,
$$

with a symmetric matrix $A(u)$ with rational entries $a_{i j}(u)$ and homogeneous coordinates $\left(x_{0}, x_{1}, x_{2}\right) \mathbb{R}$ in $\mathbb{P}^{2}$. Then there exist rational functions $y_{0}(u), y_{1}(u)$ and $y_{2}(u)$ which satisfy (8) identically for all $u$. 
Proof: This Lemma is a special case of Tsen's theorem, see [14] page 74, or [7, 9] for a more constructive version of the proof. We give here only a short outline. One way to construct a rational curve $\mathbf{y}(u)=\left(y_{0}, y_{1}, y_{2}\right)(u)$ which satisfies (8) identically, is to apply a coordinate transformation such that $A(u)$ is given in diagonal form

$$
L(u) x_{0}^{2}+M(u) x_{1}^{2}+N(u) x_{2}^{2}=0,
$$

where we can assume that the coefficients $L, M$ and $N$ are polynomials. After some preparatory steps we can assume that the polynomials $L, M$ and $N$ do not have common zeros. The zeros of these polynomials lead to necessary conditions for the solution $\mathbf{y}$. Since we are only interested in real rational parameterizations, we have to assume that $A(u)$ carries real points for all $u$. Let $u^{\star}$ be a zero of $L$. Then we obtain $M\left(u^{\star}\right) x_{1}^{2}+N\left(u^{\star}\right) x_{2}^{2}=0$, which factorizes to with possibly conjugate complex coefficients depending on the signs of $M\left(u^{\star}\right)$ and $N\left(u^{\star}\right)$. In any case we obtain linear relations for the unknown functions $x_{1}$ and $x_{2}$. Depending on the degrees of the polynomials $L, M$ and $N$ it is possible to choose polynomials $x_{0}(u), x_{1}(u)$ and $x_{2}(u)$, and their coefficients are determined by the above linear equations and possibly one quadratic equation. Finally there is at least a three-parameter family of curves satisfying (8).

According to Lemma 1 there exists a rational curve $\mathbf{x}(u)=\left(x_{0}, x_{1}, x_{2}\right)(u)$ following the family of conics $A(u)$. Applying stereographic projection to each of the conics with center $\mathbf{x}(u)$ we finally obtain a rational parameterization $\mathbf{z}(u, v)$ satisfying (8) identically, with the property that the $v$-lines of $\mathbf{z}(u, v)$ are the conics $A(u)$.

What does this mean for the equation (7)? Introducing a homogeneous parameter $v=$ $w_{2} / w_{0}$ this equation is rewritten as

$$
A(u): w_{0}^{2} \mathbf{c}^{2}+2 w_{0} w_{2} \mathbf{c} \cdot \mathbf{e}+w_{2}^{2} \mathbf{e}^{2}-w_{1}^{2}=0,
$$

where $\|\mathbf{f}(u, v)\|^{2} w_{0}^{2}$ is replaced by $w_{1}^{2}$. The scalar products of $\mathbf{c}$ and $\mathbf{e}$ are rational functions in $u$, and thus (9) can be written in the form (8). Lemma (1) tells us that there exists a rational curve $\mathbf{w}(u) \mathbb{R}=\left(w_{0}, w_{1}, w_{2}\right)(u) \mathbb{R}$ following the family of conics $A(u)$ from $(9)$. This proves

Lemma 2 Let $\mathbf{f}(u, v)=\mathbf{c}(u)+v \mathbf{e}(u)$ be a parameterization of a rational ruled surface $F$ in $\mathbb{R}^{3}$. Then there exists a rational curve $\mathbf{h}(u)=\mathbf{f}(u, v(u))$ in $F$ with rational distance $\|\mathbf{h}(u)\|$ from the origin $O=(0,0,0)$, with

$$
v(u)=\frac{w_{2}(u)}{w_{0}(u)}, \text { and }\|\mathbf{h}(u)\|=\frac{w_{1}(u)}{w_{0}(u)},
$$

where $\left(w_{0}, w_{1}, w_{2}\right)(u)$ is a rational solution of $(9)$.

Performing a stereographic projection to each conic $A(u)$ from $(9)$ we obtain a parameterization $\mathbf{w}(u, t)$ of $\mathbb{P}^{2}$ with the property that the $t$-lines of $\mathbf{w}(u, t)$ are the conics of the 
family. Letting $v=w_{2}(u, t) / w_{0}(u, t)$ and inserting this into $\mathbf{f}(u, v)=\mathbf{c}(u)+v \mathbf{e}(u)$ results in a parameterization

$$
\mathbf{f}(u, t)=\mathbf{c}(u)+\frac{w_{2}(u, t)}{w_{0}(u, t)} \mathbf{e}(u), \text { with }\|\mathbf{f}(u, t)\|=\frac{w_{1}(u, t)}{w_{0}(u, t)} .
$$

of $F$ with the property that $\|\mathbf{f}(u, t)\|$ is rational. Substituting the variable $t$ by $v$, we consequently state

Corollary 3 Let $F$ be a rational ruled surface in $\mathbb{R}^{3}$. Then there exists a rational polar representation $\mathbf{f}(u, v)=r(u, v) \mathbf{k}(u, v)$ in terms of a rational distance function $r(u, v)$ and a rational parameterization $\mathbf{k}(u, v)$ with $\|\mathbf{k}\|=1$. The straight lines on $F$ are represented by the $v$-lines of $\mathbf{f}(u, v)$.

We note that the radius function $r(u, v)$ and the the parameterization $\mathbf{k}(u, v)$ of $S^{2}$ corresponding to $\mathbf{f}(u, v)$ are

$$
r(u, v)=\|\mathbf{f}(u, v)\|, \text { and } \mathbf{k}(u, v)=\frac{1}{\|\mathbf{f}(u, v)\|} \mathbf{f}(u, v) .
$$

Consequently the conchoid $G$ of $F$ for distance $d$ with respect to $O$ is determined by

$$
\mathbf{g}(u, v)=\frac{\|\mathbf{f}(u, v)\|+d}{\|\mathbf{f}(u, v)\|} \mathbf{f}(u, v)=\frac{w_{1}+d w_{0}}{w_{0} w_{1}}\left(w_{0} \mathbf{c}(u)+w_{2} \mathbf{e}(u)\right),
$$

with $\left(w_{0}, w_{1}, w_{2}\right)(u, t)$ as parameterization of the family of conics $A(u)$ given by equation (9). The right hand side of (13) serves also to estimate degrees of the parameterization of the conchoid $G$ for a given ruled surface $F$. Finally we obtain

Theorem 4 The conchoid surface $G$ of a rational ruled surface $F$ in $\mathbb{R}^{3}$ is rational and real rational parameterizations are constructed explicitly.

\subsection{Spherical part and radius function}

To conchoid construction of a parameterized ruled surface $F$ relies on the polar representation $\mathbf{f}(u, v)=r(u, v) \mathbf{k}(u, v)$. We consider the mapping

$$
\sigma: \mathbf{f}(u, v) \rightarrow \mathbf{k}(u, v)=\sigma(\mathbf{f}(u, v))
$$

and denote $\sigma(\mathbf{f}(u, v))$ as spherical part of $F$ and $r(u, v)$ denotes $F$ 's radius function. We may write $\sigma(F)$ instead of $\sigma(\mathbf{f}(u, v))$.

Let $F$ be a general rational skew ruled surface. Its spherical part $\sigma(F)$ consists of a one parameter family of great circles in $S^{2}$ being the $v$-lines of $\mathbf{k}(u, v)$. Considering the 
parameterization $\mathbf{f}=\mathbf{c}+v \mathbf{e}$ of $F$, the circles are the intersections of $S^{2}$ with planes $E: \mathbf{x} \cdot(\mathbf{c} \times \mathbf{e})=0$. As proved in Section 2.1 there exists a rational parameterization of this family of circles. Its construction typically involves computation of roots of polynomials and is algorithmically non trivial. But there exist special cases where a rational orthonormal basis of $E$ is given, thus the spherical part $\mathbf{k}(u, v)$ of $F$ is already known. Then the computation of the radius function $r(u, v)$ becomes simpler, see for instance Section 2.6.

Typically the great circles $S^{2} \cap E$ envelope some curve being the contour of the ruled surface $F$ with respect to the central projection on $S^{2}$. This envelope curve needs not to be real and may degenerate to single points. These degenerate cases are of a certain interest.

- Let $F$ be a rational cylinder. Without loss of generality we may assume that the generating lines are parallel to $z$. That means we can choose $\mathbf{e}=(0,0,1)$. The directrix curve $\mathbf{c}(u)=\left(c_{1}, c_{2}, 0\right)$ can be chosen to be the intersection of $F$ with the plane $z=0$. The spherical part $\sigma(F)$ consists of great circles passing through the north and south pole $(0,0,1)$ and $(0,0,-1)$ of $S^{2}$, respectively. In case of rotational cylinders $F$ with $z$ as axis, the great circles are obtained by rotation. For general cylinders $\sigma(\mathbf{f}(u, v))$ can have varying speed and $\sigma(F)$ might cover only parts of $S^{2}$.

- Let $F$ be a rational cone. We assume its vertex to be $\mathbf{v}=(0,0,1)$ and choose $F \cap z=0$ as directrix curve. Since the generating lines pass through $\mathbf{v}$, the spherical part $\sigma(F)$ consists of circles passing through $(0,0,1)$ and $(0,0,-1)$. In case that $F$ is a cone of rotation with $z$ as axis, the circles are obtained by uniform rotation, otherwise the parameterization of the circles might be with varying speed and may cover only parts of $S^{2}$.

- For rational tangent surfaces $F$ of space curves $\mathbf{c}$ we have a parametric representation $\mathbf{f}(u, v)=\mathbf{c}(u)+v \dot{\mathbf{c}}(u)$. Consider the projection $\mathbf{g}(u)=\mathbf{c}(u) /\|\mathbf{c}(u)\|$ of the curve of regression $\mathbf{c}(u)$ onto $S^{2}$ with center $(0,0,0)$. The spherical part $\sigma(F)$ consists of great circles enveloping the curve $\mathbf{g}(u)$, is typically not rational.

\subsection{Distinguished directrix curve and special cases}

As discussed in Section 2.1 the computation of conchoid surfaces of rational ruled surfaces $F$ basically relies on the solution of equation (9), representing a rational family of conics in $\mathbb{P}^{2}$. For practical reasons outlined in the proof of Lemma 1 it is an advantage if $A(u)$ is a diagonal matrix.

To represent the conics of equation (9) by a diagonal matrices $A(u)$ means to represent the ruled surface $\mathbf{f}(u, v)=\mathbf{p}(u)+v \mathbf{e}(u)$, by a directrix curve $\mathbf{p}(u)$ being orthogonal to the direction vectors $\mathbf{e}(u)$. Starting from a general directrix curve $\mathbf{c}(u)$ we determine a curve $\mathbf{p}(u)=\mathbf{c}(u)+v(u) \mathbf{e}(u)$ such that $\mathbf{p} \cdot \mathbf{e}=0$ holds. This leads to $v(u)=-(\mathbf{c} \cdot \mathbf{e}) / \mathbf{e}^{2}$, and we 
obtain

$$
\mathbf{p}(u)=\mathbf{c}(u)-\frac{\mathbf{c} \cdot \mathbf{e}}{\mathbf{e} \cdot \mathbf{e}} \mathbf{e}(u) .
$$

With the foot-point curve $\mathbf{p}(u)$ of $F$ with respect to $O$, equation (9) simplifies to

$$
A(u): \mathbf{p}(u)^{2} w_{0}^{2}-w_{1}^{2}+\mathbf{e}(u)^{2} w_{2}^{2}=0 .
$$

Rational orthonormal frame of the spherical part: Remarkable special cases occur when $\|\mathbf{p}\|$ as well as $\|\mathbf{e}\|$ are rational. Without loss of generality we may assume $\|\mathbf{e}\|=1$ and we denote $\|\mathbf{p}(u)\|=\alpha(u)$ and thus there exists a spherical rational curve $\mathbf{a}(u)$ with $\mathbf{p}(u)=\alpha(u) \mathbf{a}(u)$. The spherical part $\sigma(F)$ consists of great circles being contained in planes spanned by the rational orthogonal unit vectors $\mathbf{a}(u)$ and $\mathbf{e}(u)$ and thus $\sigma(F)$ admits the parameterization

$$
\mathbf{k}(u, t)=\mathbf{a}(u) \cos t+\mathbf{e}(u) \sin t .
$$

To determine the radius function $r$, we find an obvious solution of (16) in the form $\left(w_{0}, w_{1}, w_{2}\right)(t)=(1, \alpha \cosh t, \alpha \sinh t)$ and $r(u, t)=w_{1} / w_{0}$. Converting this into a rational representation yields

$$
\left(w_{0}, w_{1}, w_{2}\right)(v)=\left(1-v^{2}, \alpha\left(1+v^{2}\right), 2 \alpha v\right), \text { and } r(u, v)=\alpha(u) \frac{1+v^{2}}{1-v^{2}} .
$$

Considering a rational curve $\mathbf{p}(u)=\alpha(u) \mathbf{a}(u)$ and a rational unit vector field $\mathbf{e}(u)$, satisfying $\|\mathbf{a}\|=\|\mathbf{e}\|=1$ and $\mathbf{a} \cdot \mathbf{e}=0$, the ruled surface $F$ is better parameterized by

$$
\mathbf{f}(u, v)=\mathbf{p}(u)+\alpha(u) \sinh v \mathbf{e}(u) .
$$

The norm $\|\mathbf{f}(u, v)\|$ equals $r(u, v)=\alpha(u) \cosh v$ and thus the conchoids $G$ of $F$ for distance $d$ can be represented in the form

$$
\mathbf{g}(u, v)=\frac{\alpha(u) \cosh v+d}{\alpha(u) \cosh v} \mathbf{f}(u, v) .
$$

Simple examples are given by the conchoids of a rotational ruled surface with respect to an axis point in Section 2.6.

\subsection{The conchoid of rational cylinders}

Let $F$ be a rational cylinder, but not necessarily rotational. According to Section 2.2 we choose the special directrix curve $\mathbf{c}(u)=\left(c_{1}, c_{2}, 0\right)(u)$, and let $\mathbf{e}=(0,0,1)$. For dealing with the general case we assume that $O \notin F$. For the squared length of $\mathbf{f}(u, v)=\mathbf{c}(u)+v \mathbf{e}$ we obtain $\|\mathbf{f}(u, v)\|^{2}=\mathbf{c}(u)^{2}+v^{2}$. We determine a reparameterization of $F$ such that $\|\mathbf{f}\|$ is rational. According to (9) the family of conics reads

$$
A(u): w_{0}^{2} \mathbf{c}(u)^{2}-w_{1}^{2}+w_{2}^{2}=0,
$$



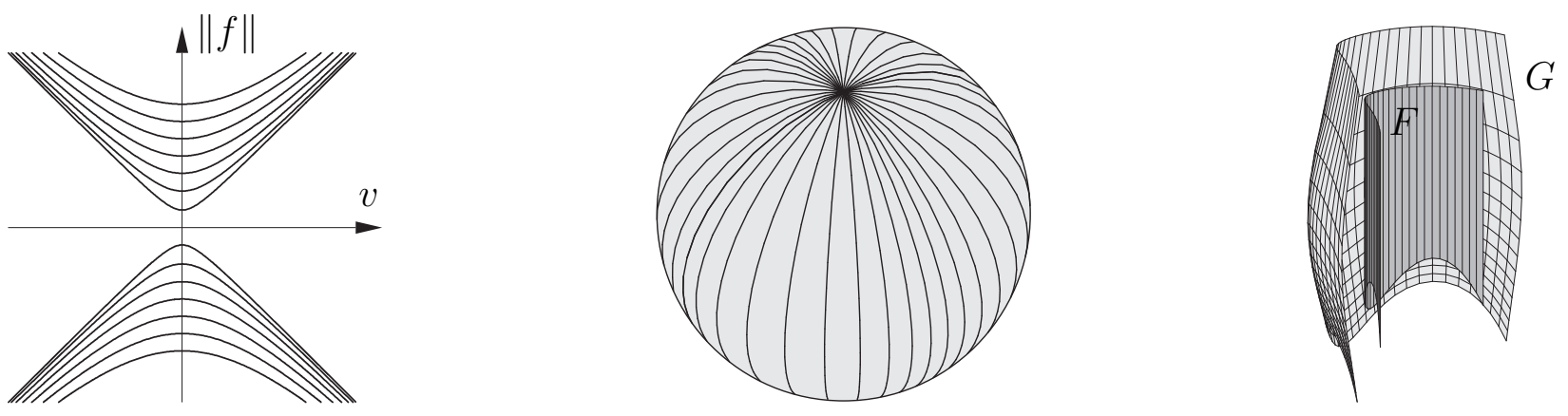

Figure 3: The family of conics (left) and the spherical part (middle) of the conchoids of a cylinder (right).

and pass through two common points $(0,1,1) \mathbb{R}$ and $(0,-1,1) \mathbb{R}$. Remembering that the spherical part $\sigma(F)$ consists of great circles through two antipodal points, the radius function has to have poles at these common points, meaning that its value is infinite. This explains that also the conics $A(u)$ share two points. Applying the stereographic projection to each of the conics $A(u)$ leads to a rational parameterization of (17),

$$
\left(w_{0}, w_{1}, w_{2}\right)(u, t)=\left(2 t, 1+\mathbf{c}(u)^{2} t^{2}, 1-\mathbf{c}(u)^{2} t^{2}\right)
$$

Substituting $v=w_{2} / w_{0}$ in $\mathbf{f}(u, v)$ leads to the rational polar representation

$$
\mathbf{f}(u, t)=\mathbf{c}(u)+\frac{1-\mathbf{c}(u)^{2} t^{2}}{2 t} \mathbf{e}, \text { with }\|\mathbf{f}(u, t)\|=\frac{w_{1}(u, t)}{w_{0}(u, t)}=\frac{1+\mathbf{c}(u)^{2} t^{2}}{2 t} .
$$

Corollary 5 Rational cylinders $F$ can be represented in a way that $\mathbf{f}(u, v)=r(u, v) \mathbf{k}(u, v)$ is a rational polar representation. This implies that the conchoid surfaces of rational cylinders admit rational parameterizations.

Explicit parameterization of the conchoids of a rational cylinder $F$ are obtained by splitting (18) into the radius function $r(u, t)$ and the spherical part $\mathbf{k}(u, t)$. With $\mathbf{f}(u, t)$ from equation (18) and $r(u, t)=\|\mathbf{f}(u, t)\|$, the conchoid surfaces $G$ of $F$ are parameterized by

$$
\mathbf{g}(u, t)=\frac{\|\mathbf{f}(u, t)\|+d}{\|\mathbf{f}(u, t)\|} \mathbf{f}(u, t) .
$$

\subsection{The conchoid of rational cones}

Let $F$ be a rational cone with vertex $\mathbf{v}=(0,0,1)$ and directrix curve $\mathbf{c}(u)=\left(c_{1}(u), c_{2}(u), 0\right)$. For dealing with the general case we assume that $O \notin F$. Then $F$ is parameterized by

$$
\mathbf{f}(u, v)=\mathbf{v}+v(\mathbf{c}(u)-\mathbf{v})=\mathbf{v}+v \mathbf{e}(u),
$$


with $\mathbf{e}(u)=\left(c_{1}(u), c_{2}(u),-1\right)$. With respect to these choices the squared length of $\mathbf{f}(u, v)$ is $\|\mathbf{f}(u, v)\|^{2}=1-2 v+\mathbf{e}(u)^{2} v^{2}$. According to (9) the family of conics determined by $\|\mathbf{f}\|^{2}$ reads

$$
A(u):-w_{1}^{2}+w_{0}^{2}-2 w_{0} w_{2}+w_{2}^{2} \mathbf{e}(u)^{2}=0,
$$

where $v=w_{2} / w_{0}$ and $w_{1}^{2}=\|\mathbf{f}\|^{2} w_{0}^{2}$. As in the case of cylinders in Section 2.4 the conics $A(u)$ share two common points $(1,-1,0) \mathbb{R}$ and $(1,1,0) \mathbb{R}$. A stereographic projection applied to the conics $A(u)$ results in a rational parameterization of $A(u)$,

$$
\left(w_{0}, w_{1}, w_{2}\right)(u, t)=\left(1-\mathbf{e}^{2} t^{2}, 1-2 t+\mathbf{e}^{2} t^{2}, 2 t(1-t)\right) .
$$

Substituting $v=w_{2} / w_{0}$ in $\mathbf{f}(u, v)$ gives the rational polar representation

$$
\mathbf{f}(u, t)=\mathbf{v}+\frac{2 t(1-t)}{1-\mathbf{e}(u)^{2} t^{2}} \mathbf{e}(u), \text { with }\|\mathbf{f}(u, t)\|=\frac{w_{1}(u, t)}{w_{0}(u, t)}=\frac{1-2 t+\mathbf{e}(u)^{2} t^{2}}{1-\mathbf{e}(u)^{2} t^{2}} .
$$

Analogously to the representation (19), an explicit rational parameterization of a conchoid $G$ of a rational cone $F$ is

$$
\mathbf{g}(u, t)=\frac{\|\mathbf{f}(u, t)\|+d}{\|\mathbf{f}(u, t)\|} \mathbf{f}(u, t)
$$

Corollary 6 Rational cones $F$ can be represented by a rational polar representation $\mathbf{f}(u, v)=$ $r(u, v) \mathbf{k}(u, v)$ which implies that their conchoid surfaces $G$ are rational.

\subsection{Conchoid surfaces of rotational ruled surfaces}

The construction of the conchoid surfaces of ruled surfaces of revolution with respect to a point on the rotational axis turns out to be elementary. Because of the rotational symmetry of the surface $F$, the computation of the conchoid $G$ of $F$ follows similar lines as for a straight line in $\mathbb{R}^{2}$. We discuss the rotational cone, the rotational cylinder and the one sheet rotational hyperboloid.

Consider a straight line $C$ in the $x z$-plane, given by the implicit equation $C: a x+b z=c$, $c \neq 0$, and $(a, b) \neq(0,0)$. We set $\mathbf{c}(v)=r(v)(\cos v, \sin v)$, with an unknown radius function $r(v)$. Plugging this parameterization into the equation of $C$ results in a linear condition for $r$, which reads

$$
r(v)=\frac{c}{a \cos v+b \sin v} .
$$

Thus the conchoid $D$ of $C$ with respect to $O$ and some chosen distance $d$ is parameterized by $\mathbf{d}(v)=(r(v)+d)(\cos v, \sin v)$. 
Cone of Rotation The radius function (23) of the line $C: a x+b z=c$ directly leads to a rational polar representation of a cone of revolution and its conchoid surfaces with respect to a point $O=(0,0,0)$ of the rotational axis $z$. Rotating $C$ around $z$, we consequently obtain

$$
\mathbf{f}(u, v)=r(v) \mathbf{k}(u, v), \text { with } \mathbf{k}=(\cos u \cos v, \sin u \cos v, \sin v),
$$

with $r(v)$ from (23). The conchoid $G$ of $F$ with respect to the distance $d$ is represented by $\mathbf{g}(u, v)=(r(v)+d) \mathbf{k}(u, v)$. It is a surface of rotation, with the conchoid $D$ of the generating line of $C$ as generating curve.

Another possibility to construct a rational polar representation of a cone of revolution is as follows. Consider the implicit equation $F: a^{2}\left(x^{2}+y^{2}\right)=(-b z+c)^{2}$ and plug in the parameterization $r(u, v) \mathbf{k}(u, v)$. For the undetermined radius function $r(u, v)$ we obtain

$$
r(u, v)=\frac{c}{ \pm a \cos v+b \sin v} .
$$

This is the radius function of the pair of lines $\pm a x+b z=c$, being the intersection of $F$ with the plane of symmetry $y=0$, and thus contains the radius function from (23).

Corollary 7 The conchoid surface of a rotational cone with respect to a point on the axis is a rotational surface with a conchoid of a line being not parallel to the axis of rotation as meridian curve.

Cylinder of Rotation Analogously to the previous case we construct the conchoid of a cylinder of rotation. We choose a line parallel to $z$ as generating line of $C$, by letting $b=0$ in the implicit equation of the line. Inserting the ansatz (24) into the equation $C: x^{2}+y^{2}=R^{2}$ of a rotational cylinder gives $r(v)= \pm R / \cos v$.

Corollary 8 The conchoid surface of a rotational cylinder with respect to a point on the axis is a rotational surface with a conchoid of a axis-parallel line as meridian curve.

Hyperboloid of Rotation Let $F$ be a one-sheet rotational hyperboloid, generated by rotating the line $(1,0,0)+v(0,1, c)$ around the $z$-axis. Its equation is $F: x^{2}+y^{2}-1 / c^{2} z^{2}=$ 1. The spherical part of $F$ consists of great circles lying in planes through $O$ and the lines of $F$. Thus the ansatz (24) does not make sense but we have to use a parameterization of $S^{2}$ based on these circles. The carrier planes of the circles are spanned by vectors

$$
\mathbf{a}=(\cos u, \sin u, 0), \text { and } \mathbf{b}=1 / g(-\sin u, \cos u, c) \text {, with } g=\sqrt{1+c^{2}} .
$$

Thus the spherical part $\sigma(F)$ of the rotational hyperboloid $F \operatorname{reads} \mathbf{k}(u, v)=\mathbf{a} \cos v+$ $\mathbf{b} \sin v$. Inserting $r(u, v) \mathbf{k}(u, v)$ with unknown radius function $r$ into the equation of $F$ gives the rational solutions

$$
r(v)=\frac{ \pm 1}{\cos v}
$$



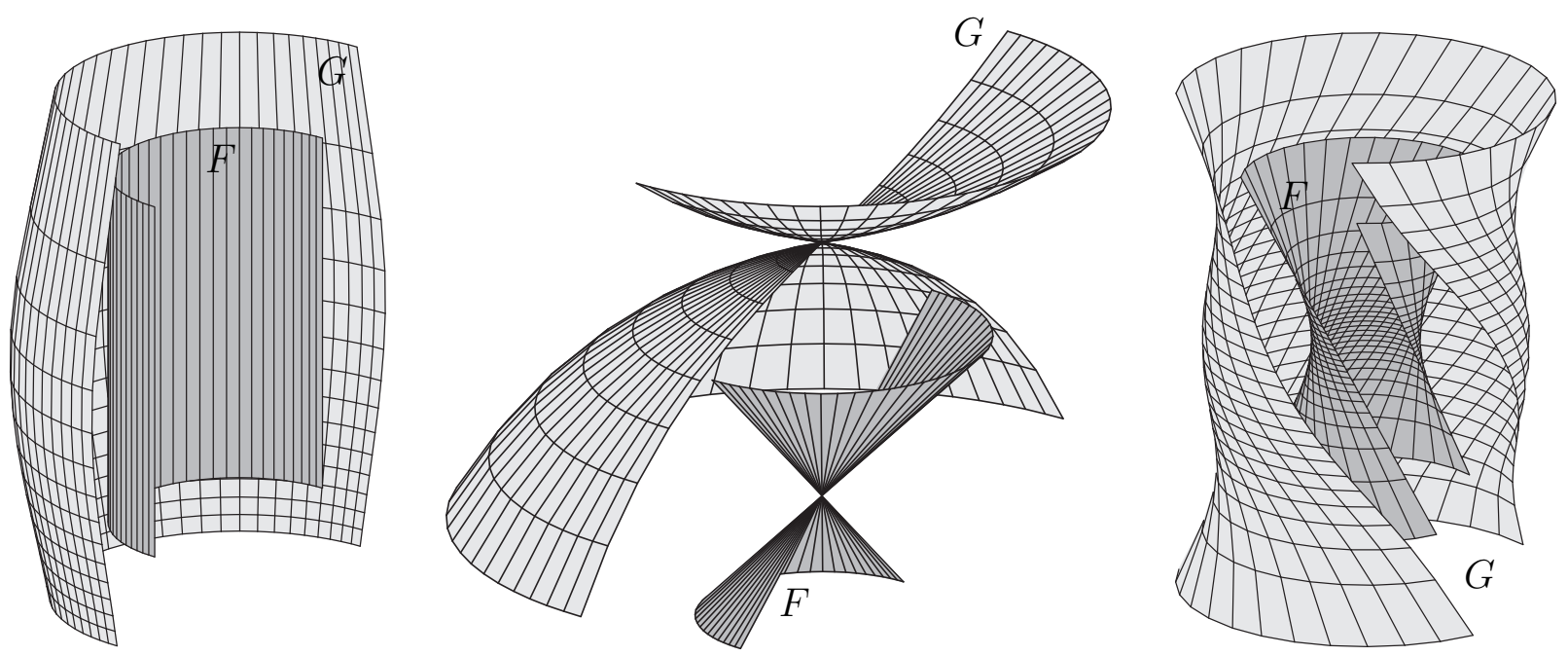

Figure 4: Rotational ruled surfaces and their conchoids with respect to an axis point. Cylinder, cone and hyperboloid.

and according to symmetry reasons $r$ does not depend on $u$. The same result is obtained when forming the family of conics (9) or (16). The squared length of the parameterization is independent on $u$ and thus leads to a family of conics with constant coefficients in the quadratic equation.

Corollary 9 The conchoid surface of a rotational hyperboloid of one sheet with respect to a point on the axis is a rotational surface with a conchoid of a line as meridian curve, where the line and the rotational axis are skew.

\subsection{The conchoid of a tangent surface of a rational space curve}

Let $F$ be the tangent surface of a rational space curve $\mathbf{c}(u)$, thus $\mathbf{f}(u, v)=\mathbf{c}(u)+v \dot{\mathbf{c}}(u)$. The curve $\mathbf{c}(u)$ is called curve of regression of $F$. For this general parameterization the squared length of $\mathbf{f}(u, v)$ is

$$
\|\mathbf{f}(u, v)\|^{2}=\mathbf{c}^{2}+2 v(\mathbf{c} \cdot \dot{\mathbf{c}})+v^{2} \dot{\mathbf{c}}^{2} .
$$

In order to obtain the normal form (16) we compute the foot-point curve $\mathbf{p}(u)=\mathbf{c}-(\mathbf{c}$. $\dot{\mathbf{c}}) /\left(\dot{\mathbf{c}}^{2}\right) \dot{\mathbf{c}}$. Then by letting $v=w_{2} / w_{0}$ the squared norm $\mathbf{f}^{2}$ simplifies to $\mathbf{p}^{2} w_{0}^{2}-w_{1}^{2}+\dot{\mathbf{c}}^{2} w_{2}^{2}=0$. In detail this reads

$$
\left(\mathbf{c}^{2} \dot{\mathbf{c}}^{2}-(\mathbf{c} \cdot \dot{\mathbf{c}})^{2}\right) w_{0}^{2}-\dot{\mathbf{c}}^{2} w_{1}^{2}+\dot{\mathbf{c}}^{4} w_{2}^{2}=0
$$

if we insert the respective expression for $\mathbf{p}(u)$. Typically the computation of a rational polar representation is analogous to the general case of skew ruled surface discussed in section 2 . 
Rational spherical curve of regression A particular case has to be discussed where the curve or regression $\mathbf{c}(u)$ is a spherical curve. Without loss of generality we assume $\|\mathbf{c}\|=1$. Then the squared length of $\mathbf{f}(u, v)$ becomes $\|\mathbf{f}(u, v)\|^{2}=1+v^{2} \dot{\mathbf{c}}^{2}$ which defines the family of conics $A(u):-w_{1}^{2}+w_{0}^{2}+\dot{\mathbf{c}}^{2} w_{2}^{2}=0$. A parameterization $\mathbf{w}(u, t)$ of $\mathbb{P}^{2}$ whose $t$-lines are the given conics $A(u)$ is

$$
\mathbf{w}(u, t)=\left(w_{0}, w_{1}, w_{2}\right)(u, t)=\left(1-\dot{\mathbf{c}}^{2} t^{2}, 1+\dot{\mathbf{c}}^{2} t^{2}, 2 t\right),
$$

and the reparameterized surface $F$ with rational radius function is given by

$$
\mathbf{f}(u, t)=\mathbf{c}(u)+\frac{2 t}{1-\dot{\mathbf{c}}^{2} t^{2}} \dot{\mathbf{c}}(u), \text { with }\|\mathbf{f}(u, t)\|=\frac{w_{1}(u, t)}{w_{0}(u, t)}=\frac{1+\dot{\mathbf{c}}^{2} t^{2}}{1-\dot{\mathbf{c}}^{2} t^{2}}
$$

The conchoids $G$ of $F$ are parameterized by (13).

\section{$3 \quad$ Examples}

This section shows five examples in detail. The conchoids of a cylinder and cone of revolution, the conchoid of a one-sheet hyperboloid, the conchoid of a hyperbolic paraboloid and finally the conchoid construction of a famous cubic ruled surface, the Plcker conoid, which is projectively equivalent to the Whitney umbrella. The necessary computational steps are outlined. To obtain a diagonal normal form of the family of conics determined by the squared distance $\left\|\mathbf{f}(u, v)^{2}\right\|$ we might use the method presented in Section 2.3. The examples here show another method. We represent the conics $A(u)$ in a coordinate system which is based on the the vertices of a polar triangle of the conics $A(u)$ as base points.

\subsection{Cylinder of revolution}

To calculate the conchoid of a cylinder of revolution $F$ with respect to the origin, one choose the generating lines of $F$ parallel to $z$, thus $\mathbf{e}=(0,0,1)$, and a circular cross section with center $(0, a, 0)$ and radius $r$ as directrix. A parametric representation of $F$ reads,

$$
\mathbf{f}(u, v)=\left(r \frac{1-u^{2}}{1+u^{2}}, r \frac{2 u}{1+u^{2}}+a, v\right) .
$$

Equation (9) reads in this case

$$
\left(\left(u^{2}+1\right)\left(r^{2}+a^{2}\right)+4 r a u\right) w_{0}^{2}-\left(u^{2}+1\right) w_{1}^{2}+\left(u^{2}+1\right) w_{2}^{2}=0 .
$$

Considering the Cartesian coordinates $\left(w_{1} / w_{0}, w_{2} / w_{0}\right)$, this defines a family of hyperbolas with common asymptotes. Their ideal points are $(0,1,1) \mathbb{R}$ and $(0,-1,1) \mathbb{R}$. The stereographic projection of the line $g(t)=(0,1, t)$ onto the hyperbolas with center $(0,-1,1) \mathbb{R}$ 
leads to a polynomial solution of (26),

$$
\begin{aligned}
& w_{0}(u, t)=-2 t\left(u^{2}+1\right) \\
& w_{1}(u, t)=-\left(r^{2}+a^{2}-t^{2}\right)\left(u^{2}+1\right)-4 a r u \\
& w_{2}(u, t)=\left(r^{2}+a^{2}-t^{2}\right)\left(u^{2}+1\right)+4 a r u
\end{aligned}
$$

Hence

$$
v(u, t)=\frac{w_{2}(u, t)}{w_{0}(u, t)}=-\frac{\left(r^{2}+a^{2}-t^{2}\right)\left(u^{2}+1\right)+4 a r u}{2 t\left(u^{2}+1\right)} .
$$

Substituting (27) in (25) leads to a rational polar representation of $F$ with

$$
\|\mathbf{f}(u, t)\|=\frac{w_{1}(u, t)}{w_{0}(u, t)}=\frac{\left(r^{2}+a^{2}-t^{2}\right)\left(u^{2}+1\right)+4 a r u}{2 t\left(u^{2}+1\right)} .
$$

A rational parameterization of the conchoids $G$ with focal point $O$ and distance $d$ reads

$$
\mathbf{g}(u, t)=\left(\begin{array}{c}
b(u, t) r\left(1-u^{2}\right) \\
b(u, t)\left(2 r u+a\left(1+u^{2}\right)\right) \\
-\frac{1}{2 t} b(u, t)\left(\left(r^{2}+a^{2}-t^{2}\right)\left(u^{2}+1\right)+4 a r u\right)
\end{array}\right)
$$

with

$$
b(u, t)=\frac{\left(u^{2}+1\right)\left(r^{2}+a^{2}+t^{2}+2 d t\right)+4 a r u}{\left(u^{2}+1\right)\left(\left(u^{2}+1\right)\left(r^{2}+a^{2}+t^{2}\right)+4 a r u\right)} .
$$

Remark: The $u$-lines of $\mathbf{g}(u, t)$ are planar and their carrying planes envelope a parabolic cylinder. The normal sections to the generating direction are parabolas with focal points that lie on the line $f(w)=(w,-1 /(2 a), 0)$, their parameter is $p=a$ and their axis are parallel to the $y$-axis.

\subsection{Cone of revolution}

A rational parameterization of a cone $F$ with vertex at $(0,2,0)$, axis in $z$-direction and an opening angle of $\pi / 2$ is given by

$$
\mathbf{f}(u, v)=\left(\frac{\left(u^{2}-1\right)(v-1)}{u^{2}+1}, \frac{2\left(u^{2}-(v-1) u+1\right)}{u^{2}+1},-v+1\right) .
$$

The family of conics $A(u)$ corresponding to $\|\mathbf{f}(u, v)\|^{2}$ reads

$$
2\left(3 u^{2}+4 u+3\right) w_{0}^{2}-\left(u^{2}+1\right) w_{1}^{2}+2\left(u^{2}+1\right) w_{2}^{2}-4(u+1)^{2} w_{0} w_{2}=0 .
$$

To obtain a diagonal normal form of the family of conics $A(u)$, we consider the polar triangle

$$
\mathbf{b}_{0}=(0,1,0), \mathbf{b}_{1}=(0,0,1), \mathbf{b}_{2}=\left(u^{2}+1,0,(u+1)^{2}\right) .
$$



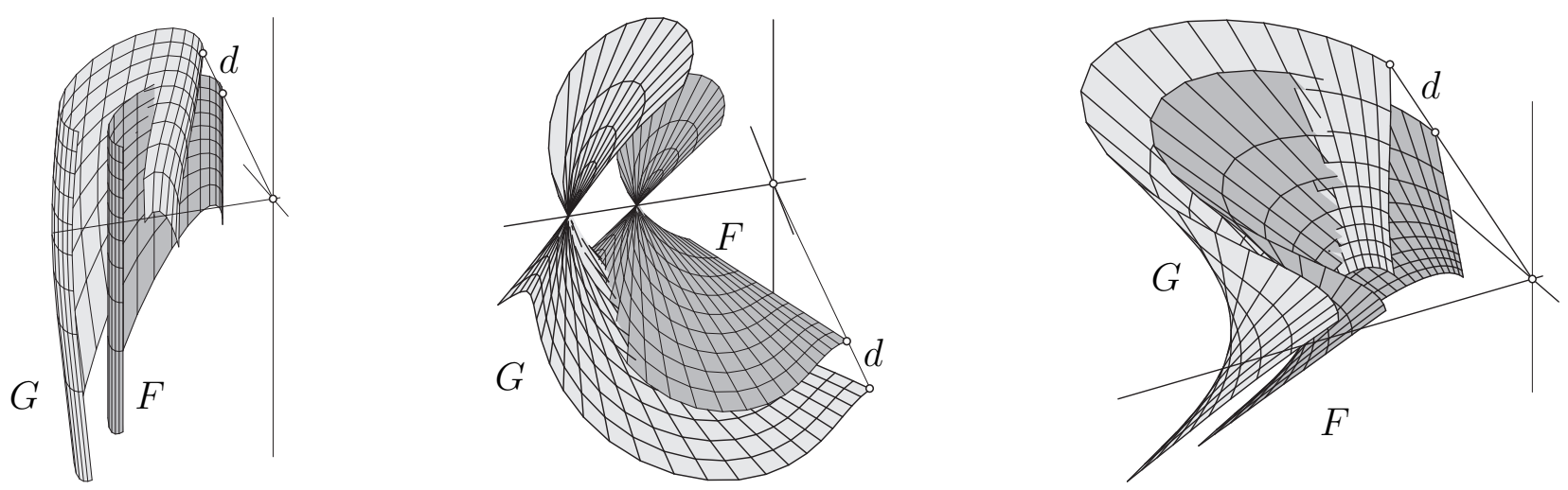

Figure 5: Conchoid $G$ of a cylinder (left), a cone (right) and a hyperboloid of revolution $F$ at distance $d=1$.

The transformation $\left(w_{0}, w_{1}, w_{2}\right)^{T}=B\left(\bar{w}_{0}, \bar{w}_{1}, \bar{w}_{2}\right)^{T}$, with $B=\left(\mathbf{b}_{0}, \mathbf{b}_{1}, \mathbf{b}_{2}\right)$ and results in a diagonal matrix $\bar{A}(u)=B^{T} \cdot A(u) \cdot B$, and the family of conics read

$$
\bar{A}(u):-\bar{w}_{0}^{2}+2 \bar{w}_{1}^{2}+4 \bar{w}_{2}^{2}\left(u^{4}+1\right)=0 .
$$

These conics share the vertices $( \pm \sqrt{2}, 1,0) \mathbb{R}$. Performing a stereographic projection of the line $(0,1, t) \mathbb{R}$ onto the hyperbolas of equation $(29)$ with center $(\sqrt{2}, 1,0) \mathbb{R}$ results in a rational parameterization $\left(w_{0}, w_{1}, w_{2}\right)(u, t)$ of $A(u)$ and the reparameterization along the generating lines of $F$ reads

$$
v(u, t)=\frac{w_{2}}{w_{0}}=\frac{-2 t^{2}\left(u^{4}+1\right)+2 t(u+1)^{2}+1}{2 t\left(u^{2}+1\right)} .
$$

Substituting (30) in (28) gives a rational polar representation of $\mathbf{f}(u, t)$ with,

$$
\|\mathbf{f}(u, t)\|=\frac{w_{1}}{w_{0}}=-\frac{\sqrt{2}\left(2 t^{2} u^{4}+2 t^{2}+1\right)}{2 t\left(u^{2}+1\right)},
$$

and the conchoid $G$ of $F$ at distance $d=1$ admits the rational parameterization

$$
\mathbf{g}(u, t)=\left(\begin{array}{c}
a(u, t)\left(2 t^{2}\left(-u^{6}+u^{4}-u^{2}+1\right)+4 t\left(u^{3}-u\right)+u^{2}-1\right) \\
2 a(u, t)\left(2 t(t u+1)\left(u^{4}+1\right)-u\right) \\
a(u, t)\left(u^{2}+1\right)\left(2 t^{2}\left(u^{4}+1\right)-4 t u-1\right)
\end{array}\right)
$$

with

$$
a(u, t)=\frac{2 t^{2}\left(u^{4}+1\right)+1-\sqrt{2} t\left(u^{2}+1\right)}{\left(2 t^{2}\left(u^{4}+1\right)+1\right) 2 t\left(u^{2}+1\right)^{2}} .
$$




\subsection{One-sheet hyperboloid}

A rational parameterization of a hyperboloid $F=\mathbf{f}(u, v)$ that has no symmetry with respect to the origin is given by

$$
\mathbf{f}(u, v)=\left(\frac{-u^{2}-2 u v+1}{u^{2}+1}, \frac{2\left(u^{2}+u+1\right)-v\left(u^{2}-1\right)}{u^{2}+1}, v\right)
$$

In this example equation (9) reads

$$
A(u):\left(5 u^{2}+8 u+5\right) w_{0}^{2}-\left(u^{2}+1\right) w_{1}^{2}+2\left(u^{2}+1\right) w_{2}^{2}-4\left(u^{2}-1\right) w_{0} w_{2}=0,
$$

with $\mathbf{w}=\left(w_{0}, w_{1}, w_{2}\right)$ as homogeneous coordinates in $\mathbb{P}^{2}$. The conics have to be turned into normal form, for example with the polar triangle

$$
\mathbf{b}_{0}=(0,1,0)^{T}, \mathbf{b}_{1}=(0,0,1)^{T}, \mathbf{b}_{2}=\left(u^{2}+1,0, u^{2}-1\right)^{T} .
$$

The transformation $\mathbf{w}^{T}=B \cdot \overline{\mathbf{w}}^{T}$, with $B=\left(\mathbf{b}_{0}, \mathbf{b}_{1}, \mathbf{b}_{2}\right)$ yields a normal form with diagonal coefficient matrix $\bar{A}(u)=B^{T} \cdot A(u) \cdot B$. The hyperbolas

$$
-\bar{w}_{0}^{2}+2 \bar{w}_{1}^{2}+a(u) \bar{w}_{2}^{2}, \text { with } a(u)=\left(u^{2}+2 u+3\right)\left(3 u^{2}+2 u+1\right),
$$

share the vertices $(\sqrt{2}, \pm 1,0) \mathbb{R}$. Applying a stereographic projection of the line $(0,1, t) \mathbb{R}$ onto the hyperbolas with center $(\sqrt{2}, 1,0) \mathbb{R}$ results in $\mathbf{w}(u, t)=\left(w_{0}, w_{1}, w_{2}\right)(u, t)$. The reparameterization along the generating lines of $F$ reads

$$
v(u, t)=\frac{w_{2}}{w_{0}}=\frac{-a(u) t^{2}+4 t\left(u^{2}-1\right)+2}{4 t\left(u^{2}+1\right)} .
$$

Substituting (33) into (31) leads to a rational polar representation of $\mathbf{f}(u, t)$ with

$$
\|\mathbf{f}(u, t)\|=\frac{w_{1}}{w_{0}}=-\frac{\sqrt{2}\left(a(u) t^{2}+2\right)}{4 t\left(u^{2}+1\right)} .
$$

The conchoid $G$ with respect to $O$ and distance $d=1$ admits the rational parameterization

$$
\mathbf{g}(u, t)=\left(\begin{array}{c}
2 b(u, t)\left(a(u) u t^{2}+\left(-u^{4}-2 u^{3}+2 u+1\right) 2 t-2 u\right) \\
b(u, t)\left(a(u)\left(u^{2}-1\right)\left(t^{2}-2\right)+\left(u^{4}+2 u^{3}+6 u^{2}+2 u+1\right) 4 t\right. \\
-b(u, t)\left(u^{2}+1\right)\left(a(u) t^{2}-\left(u^{2}-1\right) 4 t-2\right)
\end{array}\right)
$$

with

$$
b(u, t)=\frac{\left.2 \sqrt{2}\left(u^{2}+1\right) t+a(u) t^{2}+2\right)}{4\left(u^{2}+1\right)^{2} t\left(a(u) t^{2}+2\right)} .
$$




\subsection{Plücker conoid}

The Plücker conoid $F$ is an algebraic ruled surface of order three, also called cylindroid and projectively equivalent to the Whitney umbrella. A trigonometric parameterization with the double line as $z$-axis reads $(0,0, \sin 2 u)+v(\cos u, \sin u, 0)$. It can be generated in the following way. Rotate the $x$-axis around $z$ and superimpose this rotation by the translation $(0,0, \sin 2 u)$ in $z$-direction. An implicit equation of $F$ is $F: z\left(x^{2}+y^{2}\right)=2 x y$.

Since the $z$-axis is a double line of $F$, the origin is a double point and the computation of the conchoid with respect to $O$ is trivial. Thus we apply a translation by $(0,1,2)$. A rational parameterization of the translated surface which is again denoted by $F$ is given by

$$
\mathbf{f}(u, v)=\left(\frac{-\left(u^{2}-1\right) v}{u^{2}+1}, \frac{u^{2}+2 v u+1}{u^{2}+1}, \frac{2\left(u^{4}-2 u^{3}+2 u^{2}+2 u+1\right)}{\left(u^{2}+1\right)^{2}}\right)
$$

The squared length of $\mathbf{f}(u, v)$ corresponds to the family of conics

$$
\begin{aligned}
& \left(5 u^{8}-16 u^{7}+36 u^{6}-16 u^{5}-2 u^{4}+16 u^{3}+36 u^{2}+16 u+5\right) w_{0}^{2} \\
& -\left(u^{8}+4 u^{6}+6 u^{4}+4 u^{2}+1\right) w_{1}^{2}+\left(u^{8}+4 u^{6}+6 u^{4}+4 u^{2}+1\right) w_{2}^{2} \\
& +2\left(2 u^{7}+6 u^{5}+6 u^{3}+2 u\right) w_{0} w_{2}=0
\end{aligned}
$$

Representing the conics $A(u)$ with respect to the polar triangle

$$
\mathbf{b}_{0}=(0,1,0)^{T}, \mathbf{b}_{1}=(0,0,1)^{T}, \mathbf{b}_{2}=\left(u^{2}+1,0,-2 u\right)^{T} .
$$

transforms (3.4) into the normal form with diagonal coefficient matrix $B^{T} \cdot A \cdot B$, with $B=\left(\mathbf{b}_{0}, \mathbf{b}_{1}, \mathbf{b}_{2}\right)$. The family of hyperbolas

$$
\begin{gathered}
-w_{0}^{2}+w_{1}^{2}+\left(a(u)\left(u^{2}+1\right)\right) w_{2}^{2}=0, \\
a(u)=5 u^{8}-16 u^{7}+32 u^{6}-16 u^{5}-10 u^{4}+16 u^{3}+32 u^{2}+16 u+5,
\end{gathered}
$$

share the same vertices $( \pm 1,1,0) \mathbb{R}$.

A rational solution of (9) is computed by stereographic projection of the line $(0,1, t) \mathbb{R}$ onto the hyperbolas from $(3.4)$ with center $(-1,1,0) \mathbb{R}$. This leads to the reparameterization along the generating lines of $F$,

$$
v(u, t)=\frac{v_{2}}{v_{1}}=-\frac{a(u) t^{2}+\left(u^{2}+1\right)^{2}(4 t u-1)}{2\left(u^{2}+1\right)^{3} t} .
$$

Substituting (34) in (3.4) leads to a rational parameterization of $\mathbf{f}(u, t)$ and a rational parameterization of the conchoid $G$ with respect to $O$ and distance $d=1$

$$
\mathbf{g}(u, t)=\left(\begin{array}{c}
b(u, t)\left(u^{2}-1\right)\left(a(u) t^{2}+\left(u^{2}+1\right)^{2}(4 t u-1)\right) \\
b(u, t)\left(-a(u) t^{2} u+\left(u^{4}-1\right)^{2} t+\left(u^{2}+1\right) u\right) \\
4 t b(u, t)\left(u^{2}+1\right)^{2}\left(\left(u^{2}+1\right)^{2}-2\left(u^{2}+2\right) u\right)
\end{array}\right)
$$


with

$$
b(u, t)=\frac{a(u) t^{2}+2\left(u^{2}+1\right)^{3} t+\left(u^{2}+1\right)^{2}}{2 t\left(u^{2}+1\right)^{2}\left(a(u)\left(u^{2}+1\right)^{2} t^{2}+\left(u^{2}+1\right)^{4}\right)} .
$$

\subsection{Hyperbolic paraboloid}

A hyperbolic paraboloid can be given by $\mathbf{f}(u, v)=(u, v, u v+1)$, and the squared length of $\mathbf{f}(u, v)$ reads $\|\mathbf{f}(u, v)\|^{2}=\left(u^{2}+1\right) v^{2}+2 u v+\left(u^{2}+1\right)$. For the corresponding one parameter family of conics $A(u)$ we get

$$
A(u):\left(u^{2}+1\right) w_{0}^{2}-w_{1}^{2}+\left(u^{2}+1\right) w_{2}^{2}+2 u w_{0} w_{2}=0 .
$$

By the rational transformation $w_{0}=\bar{w}_{1}+\bar{w}_{2}, w_{1}=-\bar{w}_{0}, w_{2}=\bar{w}_{1}-\bar{w}_{2}$, the conics are transformed into the normal form, where we use again $w_{i}$ instead of $\bar{w}_{i}$,

$$
A(u)=w_{0}^{2}+\left(u^{2}-u+1\right) w_{1}^{2}+\left(u^{2}+u+1\right) w_{2}^{2}=0 .
$$

According to Lemma 1 there exist polynomials which satisfy (35) identically. Denote the coefficients of $w_{0}, w_{1}$ and $w_{2}$ in (35) by $L(u)=1, M(u)=u^{2}-u+1$ and $N(u)=u^{2}+u+1$, and their degrees are $2 l=0,2 m=22 n=2$. We make the ansatz

$$
w_{0}=\sum_{i=0}^{m+n} x_{i} u^{i}, w_{1}=\sum_{j=0}^{l+n} y_{j} u^{j}, w_{2}=\sum_{k=0}^{l+m} z_{k} u^{k}
$$

and evaluate (35) at the zeros $\sigma_{i}, \mu_{i}, \nu_{i}$ of $L, M$ and $N$, respectively. The solution of these four equations in the seven unknowns $x_{i}, y_{j}, z_{k}$ reads

$$
\begin{array}{ll}
x_{0}=-t_{1}-2 \sqrt{3} t_{2}+2 \sqrt{3} t_{3}, & x_{1}=3 t_{1}+2 \sqrt{3} t_{2}-4 \sqrt{3} t_{3}, \quad x_{2}=t_{1}, \\
y_{0}=t_{2}, & y_{1}=-\sqrt{3} t_{1}-2 t_{2}+3 t_{3}, \\
z_{0}=\sqrt{3} t_{1}+3 t_{2}-4 t_{3}, & z_{1}=t_{3} .
\end{array}
$$

We choose $t_{2}=1$ and $t_{3}=0$ and let $t_{1}$ such that $A(u)$ has an additional zero at $u=0$, thus $t_{1}=(2 \sqrt{2}-4 \sqrt{3}) / 5$. The curve $\mathbf{w}(u)=\left(w_{0}, w_{1}, w_{2}\right)(u)$ following the conics $A(u)$ has the parameterization

$$
\mathbf{w}(u)=\left(-\frac{\sqrt{6}+1}{5}\left(4-\sqrt{6}+u \sqrt{6}+2 u^{2}\right), \frac{2 \sqrt{6}-3}{5}, \frac{\sqrt{6}+1}{5}(-1+\sqrt{6}+2 u)\right) .
$$

Stereographic projection applied to each conic $A(u)$ finally leads to a reparameterization of $F$. The center of the stereographic projection is $\mathbf{w}(u)$ and the line which is projected to $A(u)$ is chosen by $\mathbf{q}(t)=(0,1, t) \mathbb{R}$. This leads to the rational polar representation of the hyperbolic paraboloid $F$,

$$
\mathbf{f}(u, t)=(u, v(t), u v(t)+1)
$$



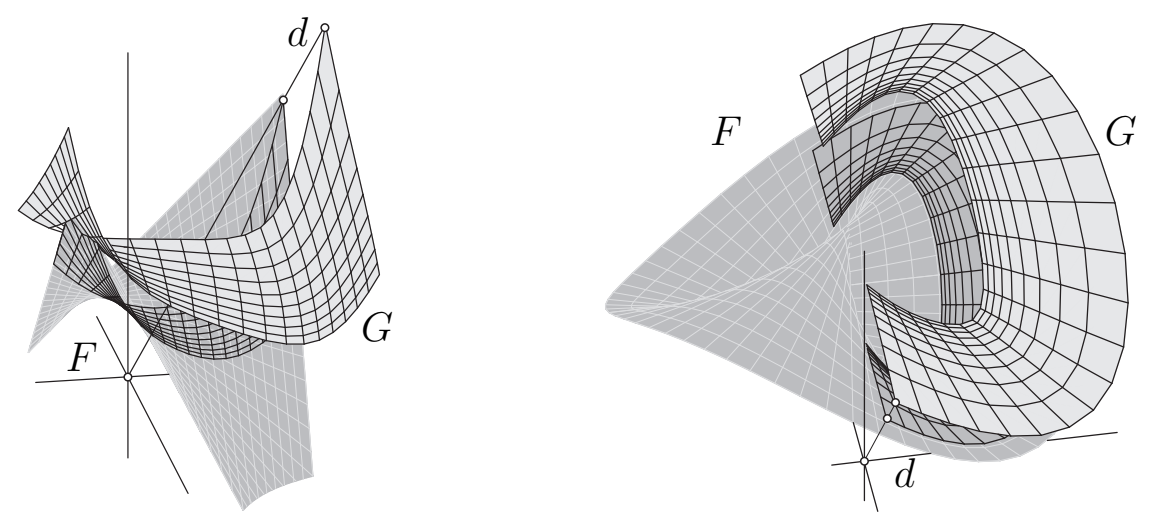

Figure 6: Conchoids $G$ of a Hyperbolic Paraboloid (left) and of a Plücker Conoid (right) at distance $d=1$.

with

$$
v(t)=\frac{b-2 t+b t^{2}+u\left(-c+2 a t-a t^{2}\right)+u^{2}\left(c-4 t-a t^{2}\right)+u^{3}\left(-1-2 t+t^{2}\right)}{-1-2 b t+t^{2}+2 u t(2+t)+2 u^{2} t(-a+t)+u^{3}\left(-1+2 t+t^{2}\right)},
$$

and the constant factors $a=1-\sqrt{6}, b=2-\sqrt{6}$ and $c=3-\sqrt{6}$. For the rational radius function of $\mathbf{f}(u, t)$ we obtain

$$
\|f(u, t)\|=\frac{\sqrt{2}\left(1+t^{2}+u\left(-1+t^{2}\right)+u^{2}\left(1+t^{2}\right)\right)\left(4-\sqrt{6}+\sqrt{6} u+2 u^{2}\right)}{2\left(-1-2 b t+t^{2}+2 u t(2+t)+2 u^{2} t(-a+t)+u^{3}\left(-1+2 t+t^{2}\right)\right)} .
$$

\section{Conclusion}

We have discussed the well known conchoid construction applied to rational ruled surfaces. The main result says that the conchoid of a rational ruled surface is a rational surface independent on the position of the focus point. The construction basically relies on the parameterization of a rational one-parameter family of conics. Special cases like cylinders, cones and rotational ruled surfaces are discussed and several examples are given to illustrate the method.

\section{Acknowledgments}

This work has been funded in parts by the Spanish 'Ministerio de Educación e Innovación' under the Project MTM2008-04699-C03-01. 


\section{References}

[1] M. Aigner, Bert Jüttler, Laureano Gonzalez-Vega, Josef Schicho, Parameterizing surfaces with certain special support functions, including offsets of quadrics and rationally supported surfaces, Journal of Symbolic Computation 44, 2009, 180-191.

[2] A. Albano, M. Roggero: Conchoidal transform of two plane curves, Applicable Algebra in Engineering, Communication and Computing, Vol.21, No.4, 2010, pp. 309-328.

[3] D. Cox D., Little J. and O'Shea D., 2010. Ideals, Varieties, and Algorithms. SpringerVerlag, New York.

[4] Krasauskas, R. and Mäurer, C., 2000. Studying cyclides with Laguerre geometry. Comp. Aided Geom. Design 17, 101-126.

[5] Lawrence, J. D., 1972. A catalog of special plane curves. Dover Publications.

[6] Peternell, M. and Pottmann, H., 1998. A Laguerre geometric approach to rational offsets, Comp. Aided Geom. Design 15, 223-249.

[7] Peternell, M., 1997. Rational Parametrizations for Envelopes of Quadric Families, Thesis, University of Technology, Vienna.

[8] Pottmann, H., 1995. Rational Curves and Surfaces with Rational Offsets, Comp. Aided Geom. Design 12, 175192.

[9] Schicho, J.,1997. Rational Parametrization of Algebraic Surfaces. Technical report no. 97-10 in RISC Report Series, University of Linz, Austria, March 1997, PhD Thesis.

[10] Schicho, J., 2000. Proper Parametrization of Real Tubular Surfaces, J. Symbolic Computation 30, 583-593.

[11] Schicho, J., 1998. Rational Parametrization of Surfaces, J. Symbolic Computation 26, $1-29$.

[12] J.R. Sendra and J. Sendra, 2008. An algebraic analysis of conchoids to algebraic curves, Applicable Algebra in Engineering, Communication and Computing, Vol.19, No.5, pp. 285-308.

[13] J. Sendra and J.R. Sendra, 2010. Rational parametrization of conchoids to algebraic curves, Applicable Algebra in Engineering, Communication and Computing, Vol.21, No.4, pp. 413-428.

[14] Shafarevich, R.I., 1994. Basic Algebraic Geometry, Vol.I, Springer, Heidelberg. 